\title{
A perioperative approach to the opioid crisis
}

\author{
Johnny-Wei Bai MD, James Bao MSPH, Anuj Bhatia MD, Vincent W.S. Chan MD
}

Cite as: CMAJ 2018 October 1;190:E1151-2. doi: 10.1503/cmaj.180801

$\mathbf{T}$ he number of opioid-related deaths continues to rise in Canada. One contributing factor that may warrant more attention is perioperative prescribing. Many patients are first exposed to opioids after surgery to relieve acute pain. This relief, however, carries the risks of adverse effects, addiction, overdose and problematic use. For some, injection heroin use a leading cause of opioid-related death - begins with nonmedical use of prescription opioids. ${ }^{1}$ Judicious prescribing and follow-up during the perioperative period has the potential to decrease opioid-related morbidity and mortality.

Guidelines recommend restricting postoperative prescriptions in duration and dose. The Institute for Clinical Systems Improvement recommends no more than three days' supply, or 20 pills, of low-dose, short-acting opioids, with re-evaluation by a physician in three to five days. ${ }^{2,3}$ Canadian and US guidelines for chronic pain also recommend limiting prescriptions to no more than 50 morphine milligram equivalents (MME) daily. ${ }^{2,4}$ Evidence, however, shows that these doses are commonly exceeded. A study of Ontario data explored opioid prescriptions for various indications. ${ }^{5}$ Among 653993 new users of prescription opioids, one in six received prescriptions for postsurgical pain, of which $40 \%$ were at doses higher than the recommended 50 MME. ${ }^{2,4,5}$ Another cohort study that included patients undergoing common surgical procedures found that patients received prescriptions of $375 \mathrm{MME}$, surpassing all recommendations for acute and chronic pain. ${ }^{2-4,6}$ It seems that despite dosing recommendations, opioids continue to be overprescribed after surgery.

The consequences of opioid overprescribing are manifold and severe. Availability of excess pills magnifies the risk of adverse effects and physiologic dependence, while contributing to tablet stockpiling, diversion into communities, intentional or unintentional overdose and opioid use disorder. Overprescribing may potentiate chronic opioid dependency; $6 \%$ of opioidnaive patients become persistent users of opioids months after surgery. ${ }^{7}$ Prolonged durations of opioid prescriptions can also predict opioid use disorder, as each additional refill after surgery increases the likelihood of misuse (dependence, abuse, overdose) by $44 \%$, and each additional week of use increases misuse by $34 \% .^{8}$ In a well-meaning effort to curb short-term postsurgical pain, clinicians may inadvertently subject their patients to long-term harm.

\section{KEY POINTS}

- Opioid overprescribing after surgery may be contributing to Canada's current opioid crisis.

- Despite guideline recommendations to limit duration and dose, evidence suggests that opioids are being prescribed for too long and at doses that are too high, after many surgical procedures.

- Overprescribing increases the risk of adverse effects, dependence, stockpiling or diversion of tablets, and opioid use disorder.

- Possible solutions include efforts to identify high-risk patients routinely, individualizing opioid prescriptions through predictive tools, improving community follow-up and leveraging specialists' roles.

Efforts are already underway to limit postoperative opioid prescribing. Enhanced Recovery After Surgery protocols emphasize multimodal analgesia and optimal nutrition to facilitate recovery. ${ }^{9}$ By de-emphasizing opioid analgesics, these protocols may decrease postoperative opioid consumption by nearly half, while decreasing pain and length of hospital stay. There are also guidelines that emphasize multimodal analgesia, including nonopioid agents, regional techniques and nonpharmacologic strategies to reduce opioid burden. ${ }^{2,10}$ Finally, implementing institution-specific prescribing guidelines sets a standard to which clinicians can be held. There is still a need, however, for novel systemic and clinical strategies to better address this problem.

Clinicians face the challenge of providing balanced analgesia after surgery: prescribing too many doses carries the risks of adverse effects and problematic use, while restrictive prescribing may result in undertreated pain, readmissions or repeated refills. This ambiguity in what comprises an "optimal opioid prescription" may bias clinicians toward providing "just-in-case" pills. The development of a tool to predict patients' opioid needs could guide prescribers to minimize opioid doses without sacrificing analgesia. This can be achieved by considering patient factors - including psychology, pain and opioid requirements in the 24 hours preceding discharge - and surgical factors, such as procedure invasiveness and whether trauma was the cause for surgery. ${ }^{11,12}$ For example, the Toronto General Hospital's 
Transitional Pain Service employs a model to predict patients at risk of high opioid requirements, problematic use and chronic postsurgical pain. Patients receive interventions to reduce opioid use and pain. ${ }^{12}$ Research to predict opioid needs could steer away from a "one-size-fits-all" approach and toward individualized prescription and harm reduction.

To further personalize opioid management, clinical tools could be used to identify patients who are at risk of adverse effects and misuse, thereby guiding targeted interventions. Scoring systems such as the Opioid Risk Tool, Brief Risk Interview, and Screener and Opioid Assessment for Patients with Pain - Revised can identify high-risk patients. ${ }^{11}$ Other predictors include anxiety, depression, catastrophizing, chronic opioid use and neuropathic pain. ${ }^{12}$ Patients with such risk factors may benefit from pain or psychiatric evaluation, multidisciplinary pain management services and multimodal analgesia. ${ }^{10}$ Clinicians and institutions should consider these predictors in their standard preoperative assessments.

Postsurgical pain and opioid management must continue after patients return to the community, ideally with re-evaluation by a primary care physician in three to five days. ${ }^{3}$ In the United States, some jurisdictions limit prescription durations to ensure patients are followed appropriately after opioid therapy is begun. More sites should involve hospital pain services, such as an acuteto-chronic pain transition service, to follow high-risk patients after discharge. When resources allow, perioperative physicians could consider limiting prescription duration with the understanding that patients receive timely pain follow-up after discharge.

Anesthesiologists and pain clinicians are well placed to manage postoperative pain in patients who are at risk of adverse outcomes. More research is needed on specific care models, but these specialists can oversee discharge prescriptions using knowledge of the patient's pain experience and opioid requirements in-hospital. This could enhance the continuum of care for patients in the postoperative period, rather than restricting the anesthesiologist's influence to the patient's time within hospital walls. Further, anesthesiologists' working relationships with surgeons can be leveraged to ensure that all prescribers adhere to recommended practices. Hospitals could facilitate communication between surgery and anesthesiology teams to optimize discharge prescriptions for high-risk patients. In the community, opportunities to refer to pain specialists may improve treatment of challenging cases of postoperative pain and opioid use.

Opioid overprescribing after surgery remains problematic despite recommendations for cautious and limited prescribing of perioperative opioids. Excess opioids place patients at risk of adverse effects and contribute to pill stockpiling, dependence and problematic use. While efforts are underway to curb overprescription, the skills of anesthesiologists and pain specialists could be leveraged to identify high-risk patients and promote conscientious prescribing and safe analgesia.

\section{References}

1. Lankenau SE, Teti M, Silva K, et al. Initiation into prescription opioid misues amongst young injection drug users. Int J Drug Policy 2012;23:37-44.

2. Busse JW, Craigie S, Juurlink DN, et al. Guideline for opioid therapy and chronic noncancer pain. CMAJ 2017;189:E659-66.

3. Thorson D, Biewen P, Bonte B, et al. Acute pain assessment and opioid prescribing protocol. 1st ed. Bloomington (MN): Institute for Clinical Systems Improvement (ICSI); 2014:1-44.

4. Dowell D, Haegerich TM, Chou R. CDC guideline for prescribing opioids for chronic pain - United States, 2016. JAMA 2016;315:1624-45.

5. Pasricha SV, Tadrous M, Khuu W, et al. Clinical indications associated with opioid initiation for pain management in Ontario, Canada: a population-based cohort study. Pain 2018;159:1562-8.

6. Thiels CA, Anderson SS, Ubl DS, et al. Wide variation and overprescription of opioids after elective surgery. Ann Surg 2017;266:564-73.

7. Brummett CM, Waljee JF, Goesling J, et al. New persistent opioid use after minor and major surgical procedures in US adults. JAMA Surg 2017;152:e170504.

8. Brat GA, Agniel D, Beam A, et al. Postsurgical prescriptions for opioid naive patients and association with overdose and misuse: retrospective cohort study. BMJ 2018;360:j5790.

9. Sarin A, Litonius ES, Naidu R, et al. Successful implementation of an Enhanced Recovery After Surgery program shortens length of stay and improves postoperative pain, and bowel and bladder function after colorectal surgery. BMC Anesthesiol 2016;16:55.

10. Chou R, Gordon DB, de Leon-Casasola OA, et al. Management of postoperative pain: a clinical practice guideline from the American Pain Society, the American Society of Regional Anesthesia and Pain Medicine, and the American Society of Anesthesiologists' Committee on Regional Anesthesia, Executive Committee, and Administrative Council. J Pain 2016;17:131-57.

11. Jones T, Lookatch S, Moore T. Validation of a new risk assessment tool: the Brief Risk Questionnaire. J Opioid Manag 2015;11:171-83.

12. Katz J, Weinrib A, Fashler SR, et al. The Toronto General Hospital Transitional Pain Service: development and implementation of a multidisciplinary program to prevent chronic postsurgical pain. J Pain Res 2015;8:695-702.

Competing interests: Vincent Chan reports receiving personal fees from Aspen Pharmacare, B. Braun, Smiths Medical and SonoSite, outside the submitted work. No other competing interests were declared.

This article has been peer reviewed.

Affiliation: Department of Anesthesia and Pain Management, Toronto Western Hospital (University Health Network), Toronto, Ont.

Contributors: All authors contributed to the conception, design and drafting of the work. Anuj Bhatia and Vincent Chan gathered studies and guidelines, applied them and mapped the order and structure of the papers. All of the authors gave final approval of the version to be published and agree to be accountable for all aspects of the work.

Correspondence to: Johnny-Wei Bai,jw.bai@mail.utoronto.ca 